Article

\title{
Two-Dimensional Transmission of Four-Dimensional LDPC-Coded Modulation with Slepian Sequences for DSP-Free 40 km Metro Network Applications
}

\author{
Xiao Han * and Ivan B. Djordjevic (D) \\ Department of Electrical and Computer Engineering, University of Arizona, 1230 E Speedway Blvd, \\ Tucson, AZ 85721, USA; ivan@arizona.edu \\ * Correspondence: xhan322@email.arizona.edu
}

check for updates

Citation: Han, X.; Djordjevic, I.B. Two-Dimensional Transmission of Four-Dimensional LDPC-Coded Modulation with Slepian Sequences for DSP-Free $40 \mathrm{~km}$ Metro Network Applications. Sensors 2022, 22, 1815 https://doi.org/10.3390/s22051815

Academic Editor: Luis Velasco

Received: 19 January 2022

Accepted: 23 February 2022

Published: 25 February 2022

Publisher's Note: MDPI stays neutral with regard to jurisdictional claims in published maps and institutional affiliations.

Copyright: (C) 2022 by the authors. Licensee MDPI, Basel, Switzerland. This article is an open access article distributed under the terms and conditions of the Creative Commons Attribution (CC BY) license (https:// creativecommons.org/licenses/by/ $4.0 /)$.

\begin{abstract}
The growing data demands are pushing researchers to pay more attention to spectrally efficient modulation formats. The four-dimensional (4D) signal constellation modulation format has been investigated for metro networks' applications to achieve better power efficiency. To cope with such modulation formats, the requirement of better digital signal processing (DSP) is also increasing rapidly. More complicated DSPs bring us extra costs; thus, the DSP-free coherent receivers are also investigated because of the high-power consumption of conventional DSP-based receivers, but the transceivers upgrading also results in extra costs. In this invited paper we implement a 4-dimentional modulation format based on Slepian sequences. We applied LDPC coding and experimentally investigated the BER performance in a two-dimensional (2D) $40 \mathrm{~km}$ fiber link transmission and demonstrate that being error free is possible without employing the complicated DSP. We compared our proposed modulation scheme with regular 16QAM and found it outperforms 16QAM with DSP over back-to-back transmission by $3.8 \mathrm{~dB}$ improvement in OSNR when BER $=10^{-5}$, while over $40 \mathrm{~km}$ metro network communication link our proposed 4D modulation signals are still successfully transmitted, and the LDPC-coding still works properly with such a new transmission strategy. On the other hand, DSP-free transmission of LDPC-coded 16-QAM exhibits an early error floor phenomenon.
\end{abstract}

Keywords: 4D modulation; Slepian sequence; DSP-free; 16QAM; metro network; 2D transmission

\section{Introduction}

The never-ending demands for higher data rates always exist in a variety of industry fields, which require significant efforts to satisfy the growing data demands and to deal with the incoming bandwidth capacity crunch [1-3], pushing researchers to pay more attention to spectrally efficient modulation formats [4]. For high data-rate transmission, the coherent communication has already become the standard communication technique in metro and long-haul networks and is becoming more and more widely applied into the datacenter space. The coherent optics has been used as a key technology for $400 \mathrm{G}$ and higher data rate transmission [5]. Quadrature amplitude modulation (QAM) formats have been investigated in [6,7] to have an excellent performance in achieving higher spectral efficiency with the same forward error correction (FEC) overhead [8]. Compared with the widely used QAM modulation formats in coherent optical communications, the more complex modulation formats, such as 4-dimensional signaling [9-12], can offer us better power efficiency, by increasing the Euclidean distance between constellation points for a given signal power when compared to the 2D signal space. The $4 \mathrm{D}$ modulation formats applications are investigated mainly for long-haul transmission [13], but have also been demonstrated to have advantages for metro networks applications in our previous work [10]. DWDM is an efficient way to increase overall capacity by allocating a huge amount of data to different wavelength channels and transmit them simultaneously [14-19]. The application of pulse shaping scheme, together with the DWDM system, can offer us 
better spectral efficiency [19]. FEC coding is an efficient method to increase the channel performance. Researchers have investigated the bit error rate (BER) performance with different codes $[20,21]$ and found the low-density parity-check (LDPC) coding outperforms other coding schemes in the high code rate region.

The capacity of DWDM optical transmission system can be increased significantly with the application of digital signal processing (DSP). To enable advanced modulation formats, the requirement of better DSP is also increasing rapidly [22]. The phase recovery algorithms for the complicated modulation formats are already investigated in [23,24], but more complicated DSPs bring us extra costs, and the conventional DSP-based receivers require high-power consumption. While the demands of different modulation formats applications are increasing, a DSP application-specific integrated circuit (ASIC) is also required to support multi-modulation and multi-rate formats [25]. Researchers have already investigated DSP-ASIC to support QAM modulation formats with different constellation points for different data rate transmissions [25-27]. The constellation shaping scheme, both probabilistic shaping (PS) and geometric shaping (GS), are broadly applied to reduce the gap of channel capacity to Shannon limit, which also require specified DSP scheme to optimize the performance [28,29]. These increasing demands of DSP costs have led researchers to pay more attention to the DSP-free coherent receivers [30-33]. On the other hand, the terabit optical Ethernet technologies will be affected by security issues, and to solve such problem, the properly designed fiber Bragg gratings (FBGs) application in impulse responses derived from mutually orthogonal Slepian sequences has been advocated in [1] to enable positive rate to convert optical communications [2,3].

In this invited paper, we apply the Slepian sequences-based basis functions to a 16points $4 \mathrm{D}$ modulation format, combined with the LDPC coding for $40 \mathrm{~km}$ metro network transmission. We find that with the application of Slepian sequences, the $4 \mathrm{D}$ modulation format can be implemented using an I/Q modulator and recovered on receiver side without any high power demanded DSP. The LDPC coding works properly with such a new modulation scheme. We experimentally demonstrate that DSP-free LDPC-coded SS-based 4D modulation scheme significantly outperforms the corresponding LDCP-coded 16-QAM counterpart in both back-to-back configuration and after $40 \mathrm{~km}$ transmission.

This invited paper is organized as follows. In Section 2 we describe the proposed SS-based LDPC-coded 4D modulation scheme. Experimental setup and experimental results are provided in Section 3. The last section summarizes the paper.

\section{Proposed Slepian Sequences-Based 16-4D LDPC-Coded Modulation Scheme}

The demands for using higher order and more complex modulation formats are increasing rapidly to satisfy never ending data requirements. To meet such transmission formats needs, more advanced DSP upgrading is required, which will bring us a large amount of implement costs. The Slepian sequence (SS), which is a discrete prolate spheroidal sequence, can offer us some new approaches to reduce DSP complexity and improve both BER performance and spectral efficiency without extra expensive hardware costs. The Slepian sequences $\left\{s_{n}^{(j)}(N, W)\right\}$ of the $j$-th order are defined as a real-valued solution to the following system of discrete Equation (1):

$$
\sum_{i=0}^{N-1} \frac{\sin 2 \pi W(n-i)}{\pi(n-i)} s_{n}^{(j)}(N, W)=\mu_{j}(N, W) s_{n}^{(j)}(N, W) ; n \in N
$$

where $i$ and $n$ denote the particular sample in each Slepian sequence, $N$ is the sequence length, and $j$ denotes the particular sequence order out of the set of sequences. The shaping factors $\mu_{j}(N, W)$ are ordered eigenvalues of the systems of Equation (1) corresponding to the concentration of each SS within the desired time interval of length $N$; thus $0<\mu_{j} \leq 1$, with 1 occurring when the sequence energy is entirely included in the desired time interval. $W$ is a discrete bandwidth $[1,3]$. 
We can easily generate the Slepian sequences of different orders, which are orthogonal to each other and as such they can be used as a new degree-of-freedom. Figure 1 shows the first 4 orders of the Slepian sequences. The Slepian Sequence we used is aimed to recover the transmitted sequence only with very simple DSP, to efficiently reduce the cost and complexity of the system.

\section{First 4 orders of Slepian sequences with 32 samples}

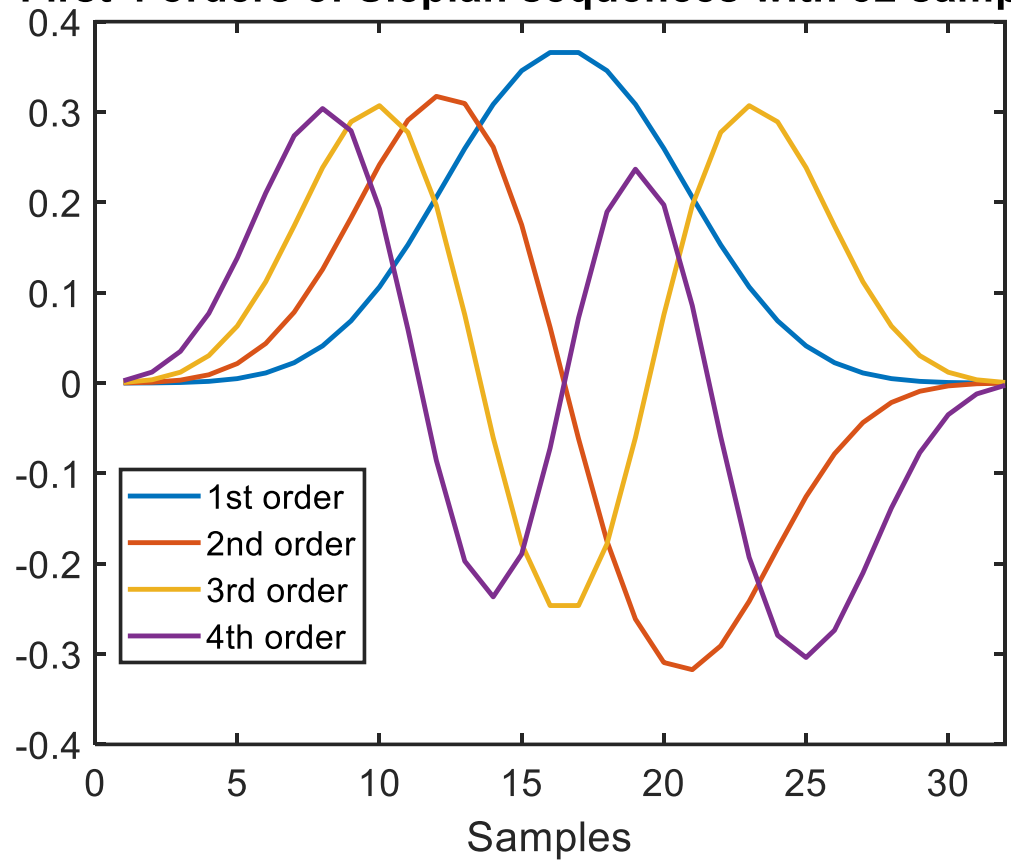

Figure 1. Different orders of Slepian sequences with 32 samples/waveform used in experimental verification.

Our proposed Slepian sequence-based 4D LDPC-coded transmitter is shown in Figure 2. A pseudorandom binary sequence (PRBS) is generated and split to 4 parallel sequences as our 4D signal. After the LDPC encoder, the encoded 4 parallel sequences are mapped to our $4 \mathrm{D}$ constellation points. The $4 \mathrm{D}$ constellation points take values of \pm 1 for every dimension. The corresponding output is then sent to the Slepian sequence mapping module, and finally we impose the 4D modulated signal on 2D I/Q modulator, by mapping the first two coordinates to the in-phase (I) channel and the last two coordinates to the quadrature $(\mathrm{Q})$ channel of an electro-optical I/Q modulator.

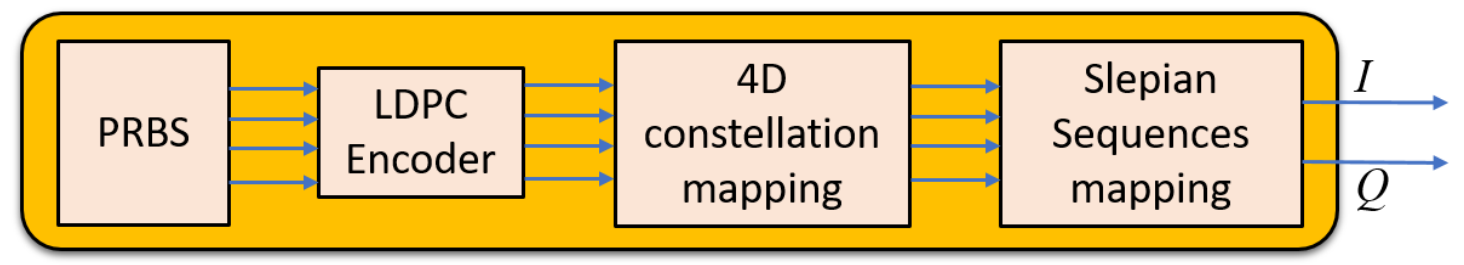

Figure 2. Slepian sequences-based 4D LDPC-coded transmitter.

As an example, to transmit a constellation point of $(+1,+1,+1,-1)$, we first map the symbol to a group of Slepian sequences:

$$
\{+(1 \text { st order }),+(2 \text { nd order }),+(3 \text { rd order }),-(4 \text { th order })\},
$$


then we have the first and second order Slepian sequences mapped to the I-channel, while the third and fourth order Slepian sequences mapped to Q-channel as follows:

$$
\left\{\begin{array}{l}
\mathrm{I}=(1 \text { st order }) \text { and }(2 \text { nd order }) \\
\mathrm{Q}=(3 \text { rd order }) \text { and }(4 \text { th order })
\end{array}\right.
$$

\section{Experimental Setup and Experimental Results}

The experimental setup is shown in Figure 3. The SS 16-4D LDPC-coded generator is introduced before in Figure 2, the output sequences are sent to a Keysight arbitrary waveform generator (AWGen) with sampling rate of $120 \mathrm{GSa} / \mathrm{s}$. Then, the analog signals are sent to an I/Q modulator. The laser source we used is a $10 \mathrm{kHz}$-linewidth, continuouswave, tunable source with center frequency of $193.4 \mathrm{THz}$. The optical signal after the I/Q modulator is amplified by an erbium-doped fiber amplifier (EDFA) of $6 \mathrm{~dB}$ noise figure. The boosted signals are coupled with the amplified spontaneous emission (ASE) noise by a $2 \times 23 \mathrm{~dB}$ coupler. We employed a variable optical attenuator (VOA) after the ASE noise source to emulate different optical SNR (OSNR) and then send the signal to the $40 \mathrm{~km}$ fiber link for the metro network communication. At the receiver side, we first applied a tunable filter (TF) to obtain the signals with our target frequency, and after amplifying the signal with EDFA the signals are sent to the coherent receiver, with the integrated coherent receiver (ICR) board and a $100 \mathrm{GS} / \mathrm{s}$ sampling rate oscilloscope from Tektronix, followed by the correlation/matched filter decision circuitry. After the correlation detector we also use the correlation approach to figure out the beginning of the LDPC-coded block (FEC frame). Then, we sent the resulting projections along the SS basis functions to the LDPC decoder to analyze the post-BER performance.

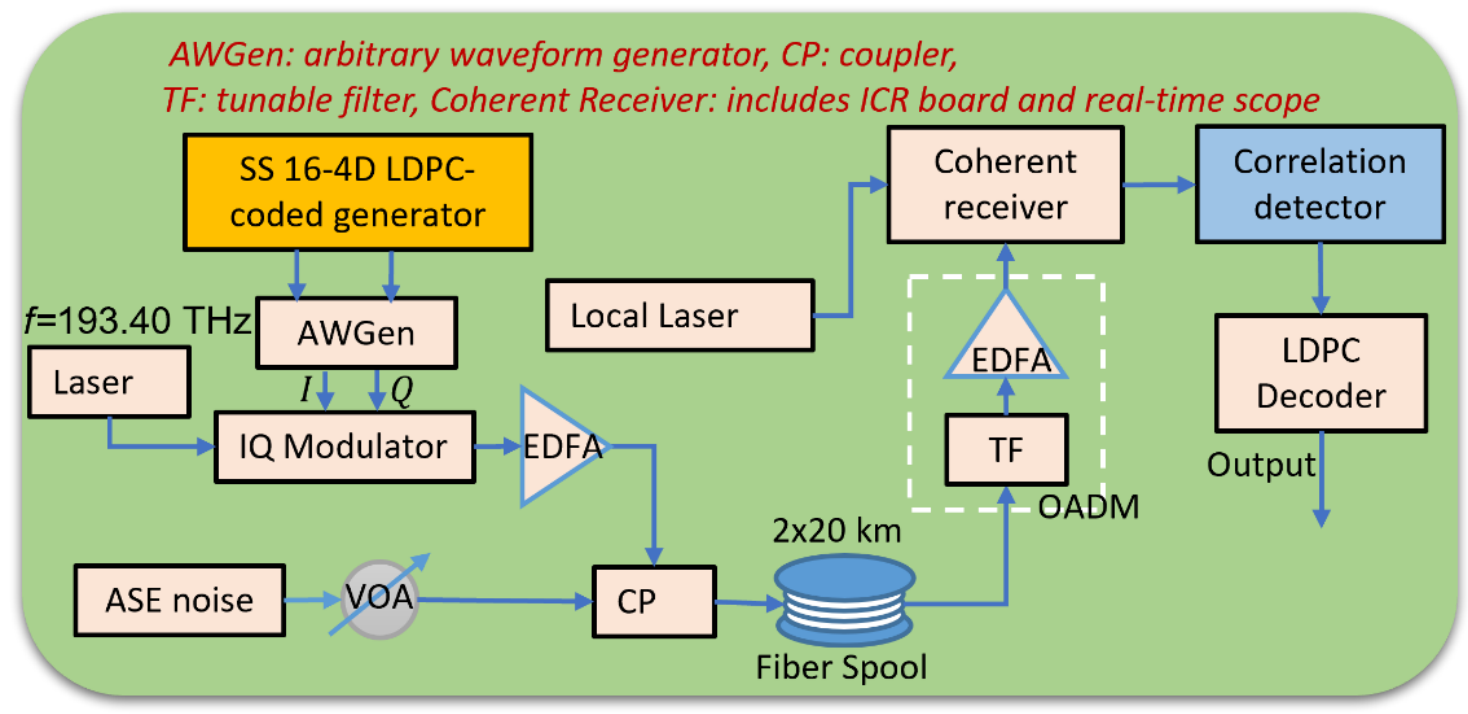

Figure 3. Experimental setup for SS-based 16-4D LDPC coded modulation scheme. OADM: optical add-drop multiplexer emulator.

The structure of our correlation detector is shown in Figure 4. Both real and imaginary parts of the received signals are split and each branch is multiplied by each order SS to calculate the correlation, followed by summation operation to implement the integration operation. We also figured out the beginning of the LDPC-coded block before the splitter. Then, we sent the resulting projections along the SS basis functions to the LDPC decoder to analyze the post-FEC BER performance. We can see the only DSP we implemented is just the correlation calculation, which strictly speaking does not belong to the DSP, and we use only 3-5 pilot symbols to perform the group sign recovery. 


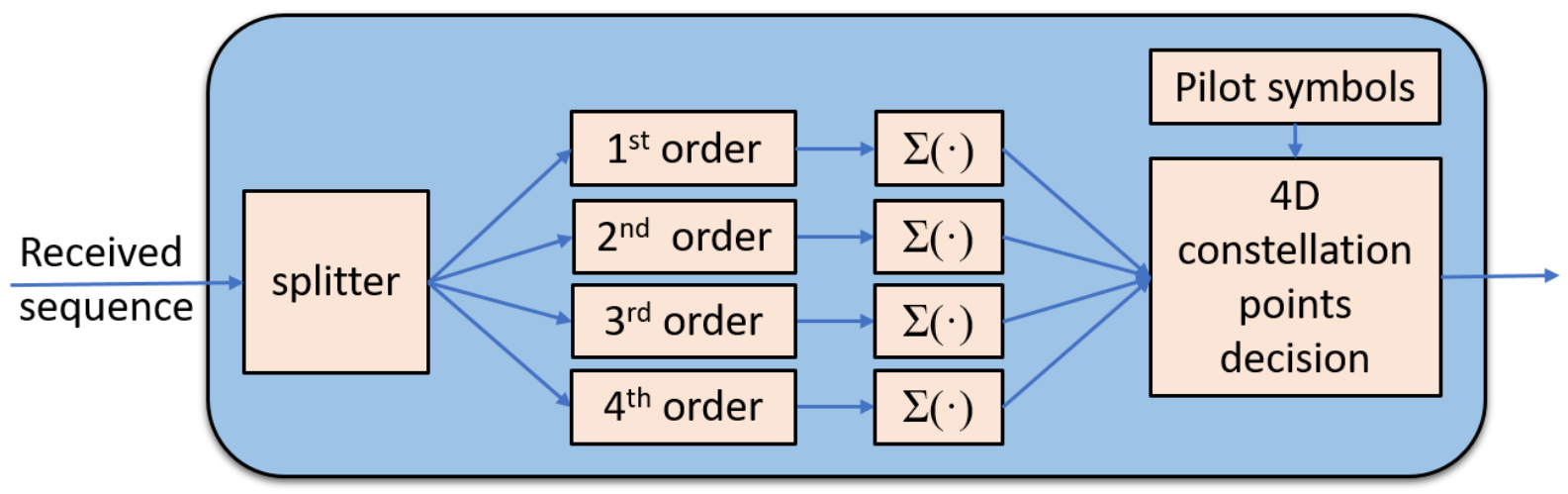

Figure 4. The configuration of the correlation detector.

The structure of our DSP progress is shown in Figure 5. The received sequences are collected from the real-time oscilloscope (Tektronics with sampling rate of $100 \mathrm{GSa} / \mathrm{s}$ ). Given that the sampling rates of AWGen and oscilloscope are different, we need to do the resampling first. After that we use the correlation calculation to do the synchronization, to find out the starting point of the codeword. The algorithm we used for the equalization is the least mean squares (LMS) algorithm. Then, we do the phase recovery, using the 4 th power algorithm. After this DSP progress, we normalized the signal and then calculate the log-likelihood ratios (LLRs) for LDPC decoding.

\section{Received}

\section{sequence}

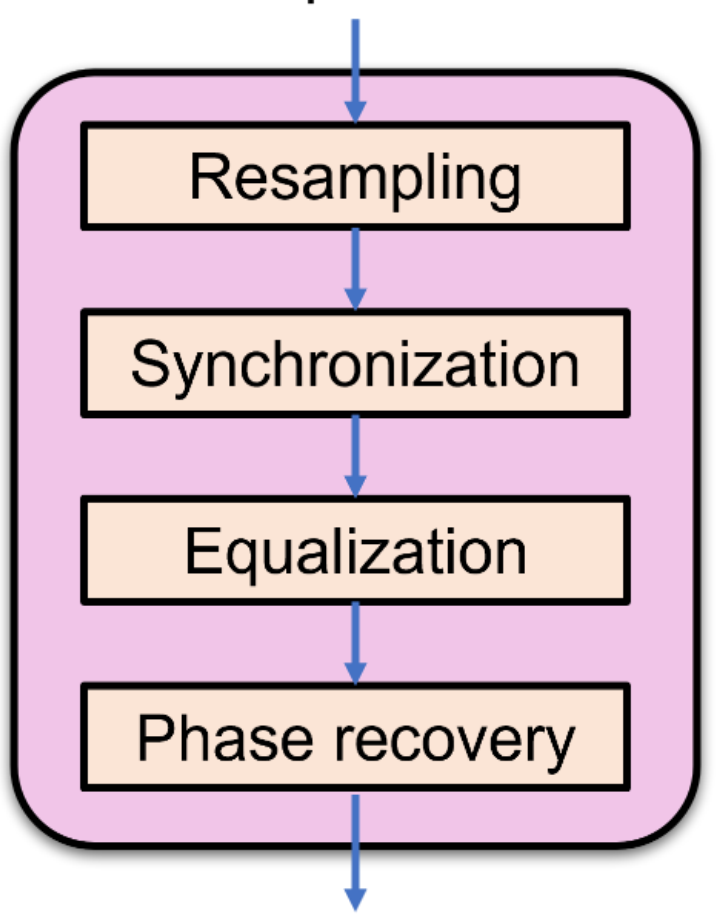

Figure 5. The configuration of the DSP progress for 16QAM.

The transmitted and received waveforms in a back-to-back (b2b) configuration are shown in Figure 6 with 250 samples. The red curve represents our generated sequence of I channel, while the blue curve is our received sequence without any noise loaded for I channel. We can see our sequence is recovered efficiently. 


\section{Waveform comparison for I channel}

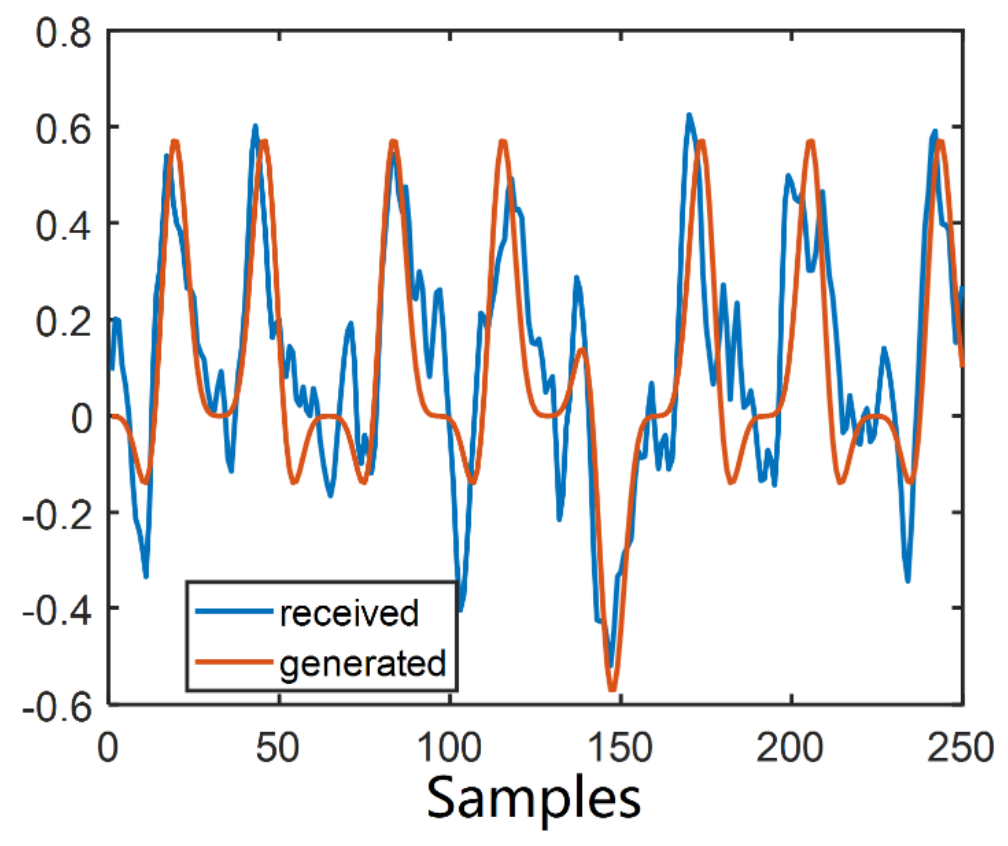

Figure 6. Comparison of received and transmitted waveforms.

The pre-FEC and post-FEC BER performances of SS-4D modulation and the traditional 16QAM are summarized in Figure 7a,b. We used both 120 GSa/s and 92 GSa/s Keysight AWGens to implement the 32 samples and 24 samples per Slepian sequence, respectively. The baud rate is $120 \mathrm{G} / 32=3.75$ GBaud for 32 samples and $92 \mathrm{G} / 24=3.833$ GBaud for 24 samples, respectively; while the baud rate of 16QAM is also 3.75 GBaud. The length of the codeword we used for both SS and 16QAM is 5648 bits, with 4239 information bits and 1409 parity check bits, thus, the code rate is 0.75 . The red curves represent the BER performance of the $4 \mathrm{D}$ modulated Slepian sequences with 32 samples; the blue curves are the BER performances of $4 \mathrm{D}$ modulated Slepian sequence with 24 samples; the cyan plots show the BER performance of regular 16QAM modulation after DSP; and non-DSP 16QAM BER performance is illustrated in the green plot. The solid plots represent the post-LDPC BER performance, while dashed curves are for pre-LDPC BER performance.

The BER performance corresponding to the back-to-back configuration is shown in Figure $7 \mathrm{a}$, we can see the proposed $4 \mathrm{D}$ modulation signals are successfully transmitted using a 4D modulator with the SS-based basis functions and the proposed LDPC-coded SSbased 4D modulation scheme significantly outperforms the regular LDPC-coded 16QAM modulation format with DSP, achieving $3.8 \mathrm{~dB}$ and $3.4 \mathrm{~dB}$ gain in OSNR when BER $=10^{-5}$ for 32 samples and 24 samples, respectively.

Figure $7 \mathrm{~b}$ shows the BER performance of different modulation formats after $40 \mathrm{~km}$ transmission. Obviously, without DSP the LDPC-coded 16-QAM exhibits early error floor and any reliable transmission is not possible. The DSP-free LDPC-code SS-based 4D modulation scheme is able to achieve error-free transmission. On the other hand, we can clearly see that the regular LDPC-coded 16QAM with DSP now outperforms the LDPCcoded DSP-free 4D SS signals for about $2.2 \mathrm{~dB}$ in OSNR at BER $=10^{-5}$, but this comparison is not fair. If we compare our results of back-to-back performance with those of $40 \mathrm{~km}$ link transmission, we can find that with the DSP, regular 16QAM does not have too much performance loss after $40 \mathrm{~km}$ transmission, but the performance of our proposed DSP-free 4D SS signal deviated a lot after $40 \mathrm{~km}$ transmission. This is because we are using the phase to carry information, but we do not apply any phase recovery. Researchers in [34-36] have already put efforts to the DSP-free transmission for simple modulation formats, such as PAM4, which is non-phase-sensitive, along with a very short transmission distance. 
If we use different orders of waveforms to transmit all information, the performance could be improved. In [36], the researchers implemented a DSP-free transmission without equalization. If we can try to apply some simple phase recovery, the performance will be promisingly improved.

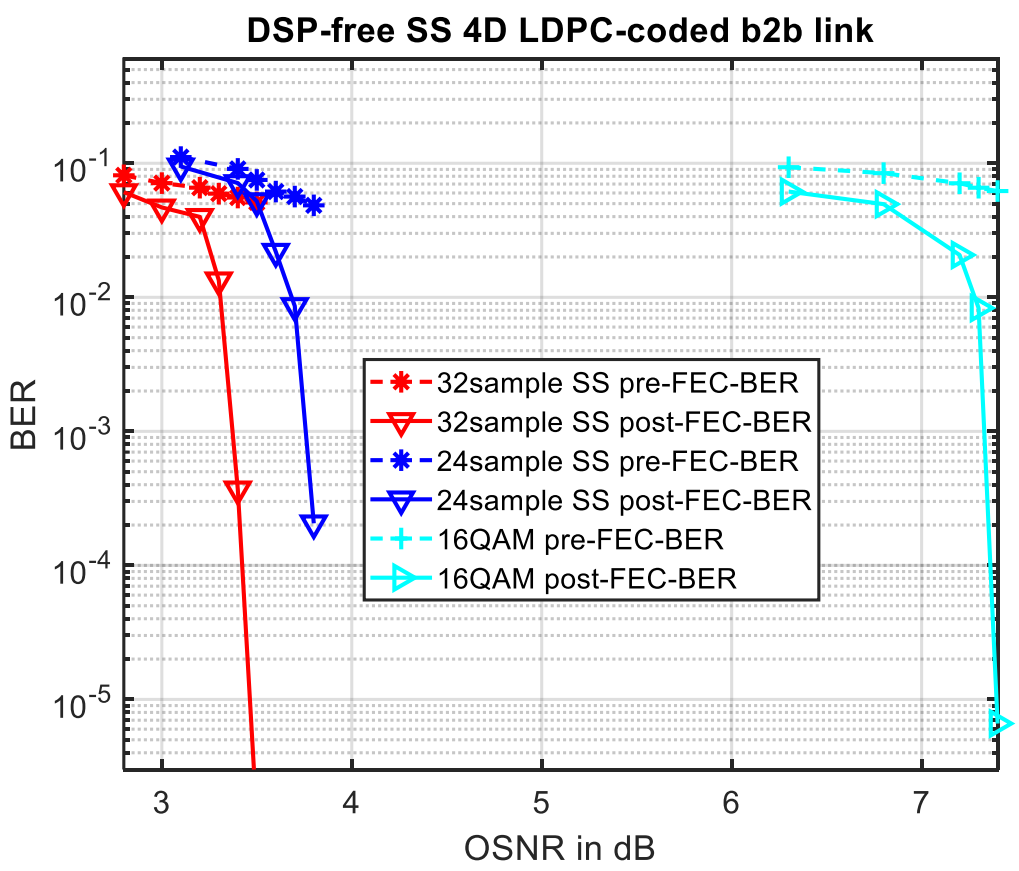

(a)

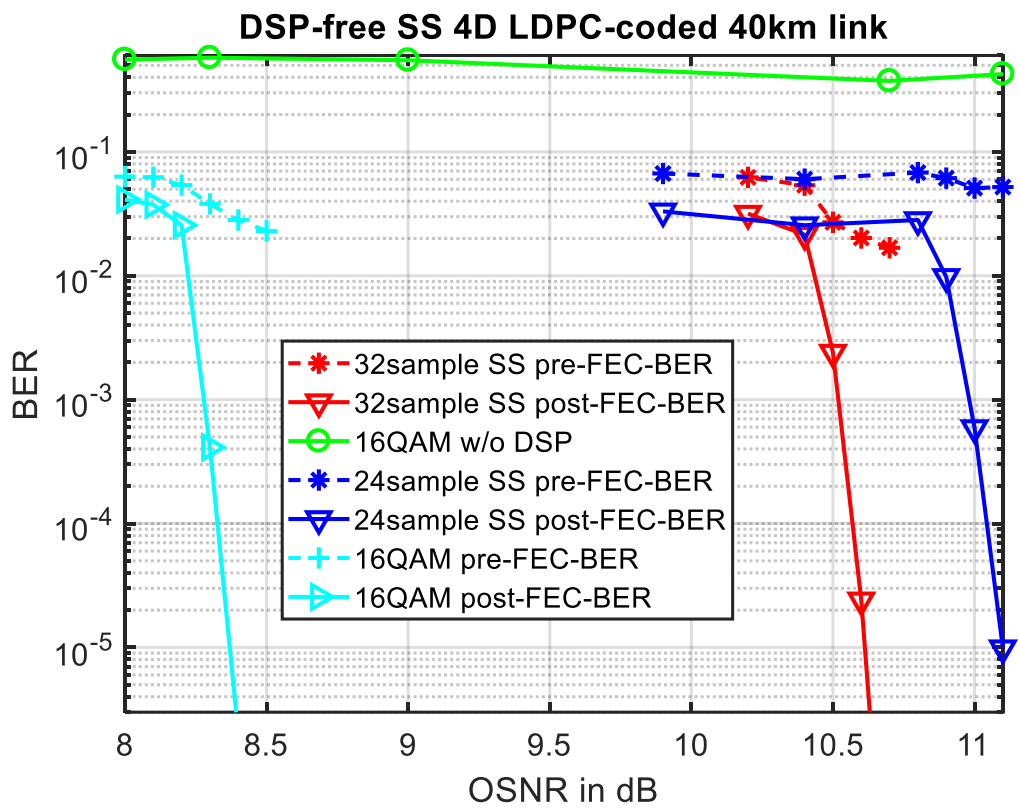

(b)

Figure 7. BER performance over OSNR for 32 and 24 samples SS 4D modulation and regular 16QAM with and without DSP: (a) in the back-to-back configuration and (b) after $40 \mathrm{~km}$ SMF link transmission.

\section{Concluding Remarks}

In this invited paper, we have applied the Slepian sequence in DSP-free 4D signaling in back-to-back configuration and in $40 \mathrm{~km}$ link transmission. We have investigated the effect of varying the number of sample points per waveform. We have compared the BER performance of the proposed SS-based LDPC-coded 4D scheme with regular LDPC- 
coded 16QAM modulation formats and find that the proposed DSP-free 4D SS signaling significantly outperforms DSP-free 16QAM modulation format in both b2b configuration and after $40 \mathrm{~km}$ transmission.

The proposed Slepian sequences-based 4D LDPC-coded modulation scheme is suitable for metro applications without any DSP. The proposed LDPC-coded SS-based 4D modulations scheme can also be used in data center applications, with results similar to Figure 7a.

To improve the baud rates instead of using AWGens to generate Slepian sequences we should fabricate the corresponding waveguide Bragg grating devices in a similar fashion as described in ref. [37].

Author Contributions: Conceptualization, I.B.D.; methodology, X.H. and I.B.D.; software, X.H.; validation, X.H.; formal analysis, X.H. and I.B.D.; investigation, X.H.; resources, I.B.D.; data curation, X.H.; writing—original draft preparation, X.H.; writing—review and editing, X.H. and I.B.D.; visualization, X.H.; supervision, I.B.D.; project administration, I.B.D.; funding acquisition, I.B.D. All authors have read and agreed to the published version of the manuscript.

Funding: This research received no external funding.

Institutional Review Board Statement: Not applicable.

Informed Consent Statement: Not applicable.

Data Availability Statement: Not applicable.

Conflicts of Interest: The authors declare no conflict of interest.

\section{References}

1. Djordjevic, I.B.; Saleh, A.H.; Küppers, F. Design of DPSS based fiber bragg gratings and their application in all-optical encryption, OCDMA, optical steganography, and orthogonal-division multiplexing. Opt. Express 2014, 22, 10882-10897. [CrossRef] [PubMed]

2. Djordjevic, I.B. Slepian-FBGs-Based Optical Covert Communications. In Proceedings of the 2018 IEEE Photonics Conference (IPC), Reston, VA, USA, 30 September-4 October 2018.

3. Djordjevic, I.B. On the energy-efficient multidimensional coded modulation for optical transport networks. In Proceedings of the 2014 IEEE Online Conference on Green Communications (OnlineGreenComm), Online, 12-14 November 2014.

4. Tan, M.; Corcoran, B.; Xu, X.; Wu, J.; Boes, A.; Nguyen, T.G.; Chu, S.T.; Little, B.E.; Morandotti, R.; Mitchell, A.; et al. Optical data transmission at 44 terabits / s with a Kerr soliton crystal microcomb. In Proceedings of the Society of Photo-Optical Instrumentation Engineers (SPIE), Online, 5 March 2021.

5. Maniloff, E.; Gareau, S.; Moyer, M. 400G and Beyond: Coherent Evolution to High-Capacity Inter Data Center Links. In Proceedings of the Optical Fiber Communication Conference (OFC), San Diego, CA, USA, 3-7 March 2019.

6. Bülow, H. Polarization QAM Modulation (POL-QAM) for Coherent Detection Schemes. In Proceedings of the Optical Fiber Communications Conference and Exhibition (OFC), San Diego, CA, USA, 22-26 March 2009.

7. Winzer, P.J.; Gnauck, A.H.; Doerr, C.R.; Magarini, M.; Buhl, L.L. Spectrally Efficient Long-Haul Optical Networking Using 112-Gb/s Polarization-Multiplexed 16-QAM. J. Lightwave Technol. 2010, 28, 547-556. [CrossRef]

8. Chen, B.; Alvarado, A.; van der Heide, S.; Hout, M.V.D.; Hafermann, H.; Okonkwo, C.M. Analysis and Experimental Demonstration of Orthant-Symmetric Four-Dimensional 7 bit/4D-Sym Modulation for Optical Fiber Communication. J. Lightwave Technol. 2021, 39, 2737-2753. [CrossRef]

9. Chen, B.; Ling, W.; Gültekin, Y.C.; Lei, Y.; Okonkwo, C.; Alvarado, A. Low-Complexity Geometrical Shaping for 4D Modulation Formats via Amplitude Coding. IEEE Photonics Technol. Lett. 2021, 33, 1419-1422. [CrossRef]

10. Han, X.; Yang, M.; Djordjevic, I.B.; Li, A. Probabilistically-Shaped Four-Dimensional LDPC-Coded Modulation in 100 km DWDM Optical Transmission for Metro Network Applications. In Proceedings of the Asia Communications and Photonics Conference (ACPC) 2019, Chengdu, China, 2-5 November 2019.

11. Cai, J.-X.; Batshon, H.G.; Mazurczyk, M.V.; Sinkin, O.V.; Wang, D.; Paskov, M.; Davidson, C.R.; Patterson, W.W.; Turukhin, A.; Bolshtyansky, M.A.; et al. $51.5 \mathrm{~Tb} / \mathrm{s}$ Capacity over 17,107 km in C+L Bandwidth Using Single-Mode Fibers and Nonlinearity Compensation. J. Lightwave Technol. 2018, 36, 2135-2141. [CrossRef]

12. Johannisson, P.; Sjödin, M.; Eriksson, T.A.; Karlsson, M. Four-Dimensional Modulation Formats for Long-Haul Transmission. In Proceedings of the Optical Fiber Communication Conference, San Diego, CA, USA, 9-13 March 2014; p. M2C.4.

13. Oliari, V.; Karanov, B.; Goossens, S.; Liga, G.; Vassilieva, O.; Kim, I.; Palacharla, P.; Okonkwo, C.; Alvarado, A. High-Cardinality Hybrid Shaping for 4D Modulation Formats in Optical Communications Optimized via End-to-End Learning. arXiv 2021, arXiv:2112.10471. 
14. Buchali, F.; Lauinger, V.; Chagnon, M.; Schuh, K.; Aref, V. 1.1 Tb/s/ $\lambda$ at 9.8 bit/s/Hz DWDM transmission over DCI distances supported by CMOS DACs. In Proceedings of the Optical Fiber Communication Conference (OFC), San Diego, CA, USA, 8-12 March 2020.

15. Rashidinejad, A.; Nguyen, A.; Olson, M.; Hand, S.; Welch, D. Real-Time Demonstration of 2.4Tbps (200Gbps/) Bidirectional Coherent DWDM-PON Enabled by Coherent Nyquist Subcarriers. In Proceedings of the Optical Fiber Communication Conference (OFC), San Diego, CA, USA, 8-12 March 2020.

16. Schadler, M.; Bocherer, G.; Pittala, F.; Calabro, S.; Stojanovic, N.; Bluemm, C.; Kuschnerov, M.; Pachnicke, S. Recurrent Neural Network Soft-Demapping for Nonlinear ISI in 800Gbit/s DWDM Coherent Optical Transmissions. J. Lightwave Technol. 2021, 39, 5278-5286. [CrossRef]

17. Han, X.; Djordjevic, I.B.; Jovanovic, A.Z. Hybrid Probabilistic-Geometric Shaped LDPC-coded PM-16QAM in 140 km DWDM Metro Network Communication. In Proceedings of the Conference on Lasers and Electro-Optics (CLEO), Online, 9-14 May 2021.

18. Chen, Y.-W.; Yi, S.; Ling, G.; West, B.; Zhao, L.; Way, W. Real-Time Demonstration of 75GHz-Spaced DWDM Single-Carrier 400G CFP2-DCO Over 1500 km. J. Lightwave Technol. 2021, 1. [CrossRef]

19. Han, X.; Yang, M.; Djordjevic, I.B.; Yue, Y.; Wang, Q.; Qu, Z.; Anderson, J. Joint Probabilistic-Nyquist Pulse Shaping for an LDPC-Coded 8-PAM Signal in DWDM Data Center Communications. Appl. Sci. 2019, 9, 4996. [CrossRef]

20. Tahir, B.; Schwarz, S.; Rupp, M. BER comparison between Convolutional, Turbo, LDPC, and Polar codes. In Proceedings of the 2017 24th International Conference on Telecommunications (ICT), Limassol, Cyprus, 3-5 May 2017.

21. Yang, F.; Fang, J.; Xiao, S.; Chen, Z.; Hu, W. Shaped polar codes for dimmable visible light communication. Opt. Commun. 2021, 496, 127126. [CrossRef]

22. Tanimura, T.; Yoshida, S.; Tajima, K.; Oda, S.; Hoshida, T. Concept and implementation study of advanced DSP-based fiberlongitudinal optical power profile monitoring toward optical network tomography. J. Opt. Commun. Netw. 2021, 13, E132-E141. [CrossRef]

23. Fatadin, I.; Ives, D.; Savory, S. Blind Equalization and Carrier Phase Recovery in a 16-QAM Optical Coherent System. J. Lightwave Technol. 2009, 27, 3042-3049. [CrossRef]

24. Civelli, S.; Forestieri, E.; Secondini, M. Interplay of Probabilistic Shaping and Carrier Phase Recovery for Nonlinearity Mitigation. In Proceedings of the 2020 European Conference on Optical Communications (ECOC), Online, 6-10 December 2020.

25. Maeda, H.; Saito, K.; Kawahara, H.; Seki, T.; Sasai, T.; Hamaoka, F. High Spectral Efficiency Real-Time 500-Gb/s/carrier Long-Haul Transmission Over Field-Installed Fibers. J. Lightwave Technol. 2021, 39, 933-939. [CrossRef]

26. Maeda, H.; Saito, K.; Sasai, T.; Hamaoka, F.; Kawahara, H.; Seki, T.; Kawasaki, T.; Kani, J.-I. Real-time 400 Gbps/carrier WDM transmission over 2,000 km of field-installed G.654.E fiber. Opt. Express 2020, 28, 1640-1646. [CrossRef]

27. Hamaoka, F.; Sasai, T.; Saito, K.; Kobayashi, T.; Matsushita, A.; Taniguchi, H.; Kuwahara, S.; Kawahara, H.; Seki, T.; Ozaki, J.; et al Dual-Carrier 1- Tb/s transmission over field-installed G.654.E fiber link using real-time transponder. IEICE Trans. Commun. 2020, E103-B, 1183-1189. [CrossRef]

28. Sun, H.; Torbatian, M.; Karimi, M.; Maher, R.; Thomson, S.; Tehrani, M.; Gao, Y.; Kumpera, A.; Soliman, G.; Kakkar, A.; et al. 800G DSP ASIC Design Using Probabilistic Shaping and Digital Sub-Carrier Multiplexing. J. Lightwave Technol. 2020, 38, 4744-4756. [CrossRef]

29. Mello, D.A.A.; Barbosa, F.A.; Reis, J.D. Interplay of Probabilistic Shaping and the Blind Phase Search Algorithm. J. Lightwave Technol. 2018, 36, 5096-5105. [CrossRef]

30. Perin, J.K.; Shastri, A.; Kahn, J.M. Design of Low-Power DSP-Free Coherent Receivers for Data Center Links. J. Lightwave Technol. 2017, 35, 4650-4662. [CrossRef]

31. Morsy-Osman, M.; Sowailem, M.; El-Fiky, E.; Goodwill, T.; Hoang, T.; Lessard, S.; Plant, D.V. DSP-free 'coherent-lite' transceiver for next generation single wavelength optical intra-datacenter interconnects. Opt. Express 2018, 26, 8890-8903. [CrossRef]

32. Ciaramella, E. Assessment of a Polarization-Independent DSP-Free Coherent Receiver for Intensity-Modulated Signals. J Lightwave Technol. 2019, 38, 676-683. [CrossRef]

33. Kamran, R.; Nambath, N.; Manikandan, S.; Ashok, R.; Jain, R.; Thaker, N.B.; Gupta, S. Demonstration of a low-power, localoscillator-less, and DSP-free coherent receiver for data center interconnects. Appl. Opt. 2020, 59, 2031-2041. [CrossRef]

34. Verbist, J.; Lambrecht, J.; Verplaetse, M.; Van Kerrebrouck, J.; Srinivasan, A.; De Heyn, P.; De Keulenaer, T.; Yin, X.; Torfs, G.; Van Campenhout, J.; et al. DAC-Less and DSP-Free 112 Gb/s PAM-4 Transmitter Using Two Parallel Electroabsorption Modulators. J. Lightwave Technol. 2018, 36, 1281-1286. [CrossRef]

35. Saber, G.; Morsy-Osman, M.; Hui, M.; El-Fiky, E.; Vall-Llosera, G.; Dortschy, B.; Urban, P.J.; Cavaliere, F.; Lessard, S.; Plant, D.V. DSP-Free 25-Gbit/s PAM-4 Transmission Using 10G Transmitter and Coherent Amplification. IEEE Photonics Technol. Lett. 2018, 30, 1547-1550. [CrossRef]

36. Rannello, M.; Presi, M.; Ciaramella, E. Optical vs. Electrical Duobinary Coding for 25 Gb/s PONs based on DSP-free Coherent Envelope Detection. In Proceedings of the Optical Fiber Communication Conference (OFC), San Diego, CA, USA, 11-15 March 2018.

37. Djordjevic, I.B. Proposal for Slepian-States-Based DV- and CV-QKD Schemes Suitable for Implementation in Integrated Photonics Platforms. IEEE Photonics J. 2019, 11, 1-12. [CrossRef] 